\title{
Evaluation of Anti-Fungal Activity of Chitosan and Its Effect on the Moisture Absorption and Organoleptic Characteristics of Pistachio Nuts
}

\author{
Atefe Maghsoudlou, Yahya Maghsoudlou, Morteza Khomeiri, Mohamad Ghorbani \\ Dept. of FST., College of Food Tech., Gorgan University Of Agricultural Sciences And Natural Resources, Iran \\ E-mail:a_maghsoodloo2000@yahoo.com,ymaghsoudlou@yahoo.com; mkhomeiri@yahoo.com; treatment@yahoo.com
}

\begin{abstract}
Pistachio is one of the main export products of Iran and Iran is one of the largest producer and exporter of pistachio in the world. Unfavourable environmental conditions during storage, causes a sharp drop in quality of product through musty and toxin production, especially aflatoxin by Aspergillus flavus and Aspergillus parasiticus, Absorption of foreign odors and moisture, Tissue destruction of undesirable flavour. The aim of this study was to study the anti-fungal activity of chitosan and its effect on the organoleptic characteristics of pistachio nuts. Therefore, using acetic acid $1 \% \mathrm{~V} / \mathrm{V}$, chitosan concentrations of $0.5 \%, 1 \%$ and $1.5 \%$ V/W was prepared and pistachios were coated by these solutions. Also acetic acid at concentration $1 \%$ without chitosan was used as a treatment for coating to determine the antimicrobial effect of acetic acid. The results showed that chitosan significantly $(p<0.05)$ inhibited the growth of the Aspergillus and its effect was increased with increased concentration. Chitosan also prevented moisture absorption and weight change in pistachio nuts, while chitosan concentration showed no significant effect on moisture absorption and weight change of pistachio nuts. Chitosan $1.5 \%$ had a significant effect $(p<0.05)$ on the flavour of pistachio, but other concentrations had no effect. However, chitosan in general had no significant effect $(p<0.05)$ on color, texture and acceptability of pistachio nuts.
\end{abstract}

\section{INTRODUCTION}

Pistachio (Pistacia Vera) belonging to Anacardiaceae family is one of the major export products of Iran, being one of the largest producer and exporter of pistachio nut in the world. Under adverse environmental conditions during storage, mold and toxin production especially Aflatoxins by Aspergillus flavus and Aspergillus parasiticus, moisture absorption, texture deterioration and rancidity, drastically reduces the product quality [1]. One method to avoid quality deterioration is use of edible coating, acting as a barrier against moisture and oxygen transfer and dissolved substances in food; while it is easily consumable. Edible coatings prevent moisture absorption in dried fruits and products, and loss of moisture in fresh fruits and meat products. Chitosan which is produced from crab and shrimp waste is used as edible film [2]. Coating valuable food products such as pistachio nut by chitosan - with high nutritional values - leads to production of functional food which would have positive effects on its nutritional values and its export, in addition it reduces the mold growth thus preventing possibility of aflatoxin production during storage period. Chitosan is a colorless and odorless copolymer of 2amino-2-deoxy-D-glucose (glucosamine), 2-acetamido-2-
deoxy-D-glucose ( $\mathrm{N}$-acetyl glucosamine) which is made by Chitin N-Deasetylasion from crab and shrimp wastes. Antibacterial effects of chitosan was first investigated and documented by Mozarely et al. in 1990 [3]. Campalnilo et al. in 2008 investigated antibacterial effect of chitosan coating on strawberry. They immersed strawberry slices in $1 \%$ Chitosan solution and packed them in plastic film. The results showed that the chitosan coatings prevented microbial growth and reduced fruit weight changes. Results of the organoleptic evaluation showed that chitosan coating had no effect on fruits flavor and general acceptability and preserved the fruit color during storage [4].

Khalid Ziani et al., in 2010 studied effect of chitosan coating on artichoke seeds preservation, where chitosan coating was used as anti-fungal agent. Research showed all chitosan treatments reduced the activity of various fungi and plants growth was increased [5]. Xianghong et al. in 2010 studied the antibacterial effect of chitosan on growth of Alternaria kikuchiana and Physalospora piricola on pear. They used $1 \%$ chitosan to coat pears. After 5 days of storage at $250 \mathrm{C}$ the antifungal effect of chitosan was dominant. Chitosan had no significant effect on the organoleptic characteristics of pears [6]. 
Monez et al. in 2009 found antifungal effect of chitosan on Colletortichum growth on tomato and berry during incubation. Increasing chitosan concentration significantly increased the antifungal effectivity of chitosan [7].

In 2007 Chein et al. examined effects of chitosan coating on quality and shelf life of mango pieces. Mango pieces were treated by $0 \%, 0.5 \%, 1 \%$ and $2 \%$ chitosan solution. Chitosan postponed weight loss, quality deterioration and prevented microbial growth. Results showed increase in chitosan concentration would not affect the antifungal activities [8]. Devlieghere et al., in 2004 studied antimicrobial effect of commercial chitosan against a variety of food-born Psychrofilic pathogens and spoilage microorganisms in lettuce and strawberries. They mentioned that antimicrobial effect of chitosan solution and lactic acid was prominent; however, it may not be applicable due to the development of bitter taste in lettuce [9].

Dong et al. in 2003 studied the effect of chitosan coating on quality conservation and shelf life of peeled litchi. They used $1 \%$ chitosan solution as coating followed by packing in plastic films at $-10 \mathrm{C}$ temperature. Chitosan application leads to delay in weight change, quality deterioration and organoleptic characteristics and effectively reduced microbial population followed by expansion shelf life [10]. In 2009, Cao et al. investigated the changes on oyster microbial flora during storage at refrigeration temperature and effect of chitosan on its shelf life at $5 \pm 10 \mathrm{C}$. In this investigation oyster mushrooms were immersed in chitosan solutions at different concentrations after preparation. Results showed that chitosan caused reduction in microbial population and its effect increased by increase in concentration. It also prevented the growth of some bacterias and $\mathrm{pH}$ reduction in edible oysters during storage. In general, oyster shelf life increased from 8-9 days to 14-15 days. Chitosan effect on the oyster organoleptic characteristics such as flavor and color were insignificant during storage period [11].

The objective of this study is to investigate the anti-fungal effect of chitosan and its effect on the organoleptic characteristics and moisture absorption of Pistachio nut.

\section{MATERIAL AND METHODS}

\section{A. Materials}

For this study, Pistachio Akbari cultivar (common commercial variety of Iran) was selected. Pistachios were provided in September 2010 from an agricultural research center in Rafsanjan. Chitosan powder purchased from Sigmaaldrich Company. Acetic acid 1\% V/V and medium culture YGC used in this study were purchased from Merck Company.

\section{B. Methods}

Pistachios were peeled, hard shells removed and wholesome nuts were selected. For coating the pistachio nuts, chitosan solutions at concentrations 0.5 , 1and $1.5 \%$ were prepared by disolving $0.5,1$ and 1.5 gr chitosan powder in acetic acid $1 \% \mathrm{~V} / \mathrm{V}$ respectively making final volume to $1000 \mathrm{CC}$. To determine the anti-microbial effect of acetic acid, $1 \%$ of acetic acid was used alone for coating as control.
The solutions prepare were then filtered and autoclaved $[12,13]$.

Pistachio nuts after weighting were placed in mesh container and immersed in chitosan solutions for 30 to 40 seconds. Coated nuts were then dried in oven at $45^{\circ} \mathrm{C}$ for 3 hours and then packed in 50 grams polyethylene bags and kept at room temperature for 6 months. During storage, sampls were examined every two weeks for total mold and yeast count, moisture content and weight changes using AOAC methods.

For measuring percent of Aspergillus development each 10 treated pistachio nuts were placed in pre-sterilises glass container plates containing moist filter paper and then incubated at $25^{\circ} \mathrm{C}$. After 3-5 days the number of infected pistachios in each plate were counted and mold expansion compared with control was expressed as percentage.

To make sure the mold growth on the pistachio nuts, slide cultures were provided and observed on a microscope to ensure the growth of Aspergillus species. However; sensory evaluation was also performed with 5 point Hedonic scale ( $1=$ bad, $2=$ poor, $3=$ mean, $4=$ good, 5=very good). In this experiment 8 people were selected from students aged 20 to 30 years as panelist, and each panelist was given 4 pistachio nut of any treatment within a transparent color container with the three-digit codes for separation at room temperature. Four factors: color, flavor, texture and general acceptability of the products were evaluated by panelist. All experiments were done in the laboratory of Food Microbiology \& Food Analysis, Faculty of Food Technology, University of Agricultural Sciences \& Natural Resources of Gorgan.

\section{Statistical analyses}

Design of experiments were factorial form in a completely randomized design in three replications. Statistical analysis is done by using the SAS software and avereges compared by using Duncan's multiple range test at $\alpha=0.05$. Excel software was used for drawing diagrams.

\section{RESULTS AND DISCUSSION}

\section{A. The antifungal activity of Chitosan}

With coating of plant products with chitosan, chitosan dual functional effect on the product shelf life: direct effect on fungi growth and different function of the immunity such as chitinase retention which reduces the fungal cell wall synthesis of proteinase inhibitors. These systems are more effective at presence of microbial transport systems [14].

1) Total mold and yeast counts: As it is shown in the figure 1, the amount of mold growth during storage in control samples is highly increased; while the antimicrobial effect of acetic acid 1\% was significant. In the sample containing edible chitosan coating, the mold growth is almost zero for the entire period [5]. There was insignificant difference in antimicrobial effects of various concentrations of chitosan same results were reported by China et al. in 2007 [14].

2) Development of Aspergillus molds: In figure 2, the effect of chitosan edible coatings to prevent the development of Aspergillus molds in pistachio is clearly visible [4].

Development of mold in the control samples reached to $100 \%$ after 5 months while in the samples containing 
chitosan edible coatings, with increasing chitosan concentration, the mold development was decreased, in $1.5 \%$ chitosan concentration, this amount is zero which coincides with Monez et al. results in 2009 [7].

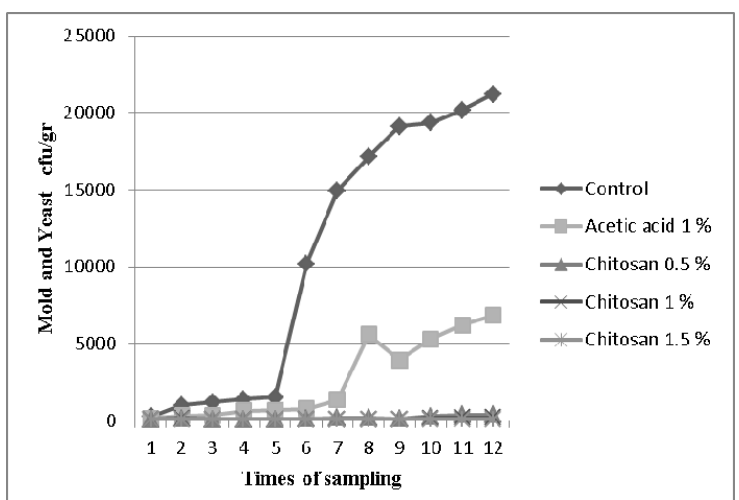

Fig 1. Chart of changes in yeasts and molds growth in pistachio nuts during 6 month storage.



Fig 2. Chart of development of Aspergillus molds in pistachio nuts during 6 month storage.

\section{B. Chitosan effect on weight change and moisture content of products}

Moisture content is an important factor in dried food quality. Moisture transfer between food and surrounding atmosphere, decreases as foods cover up with edible coating [15]. As can be seen in Fig. 3 \& 4, weight change and moisture content in the samples without chitosan coating is more than samples with coating. This is due to the effect of chitosan coating preventing moisture transfer to the pistachio nuts, which is consistent with Chein et al, results in 2007 [8].

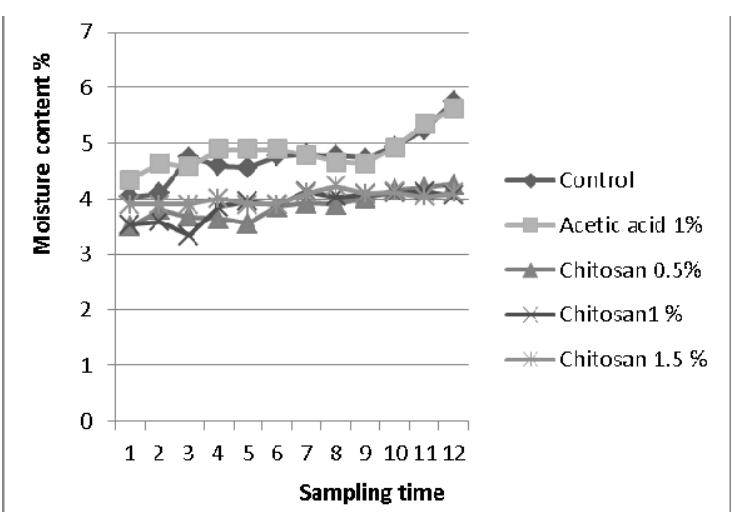

Fig 3. Chart of changes in moisture content of pistachio nuts during 6 month storage.

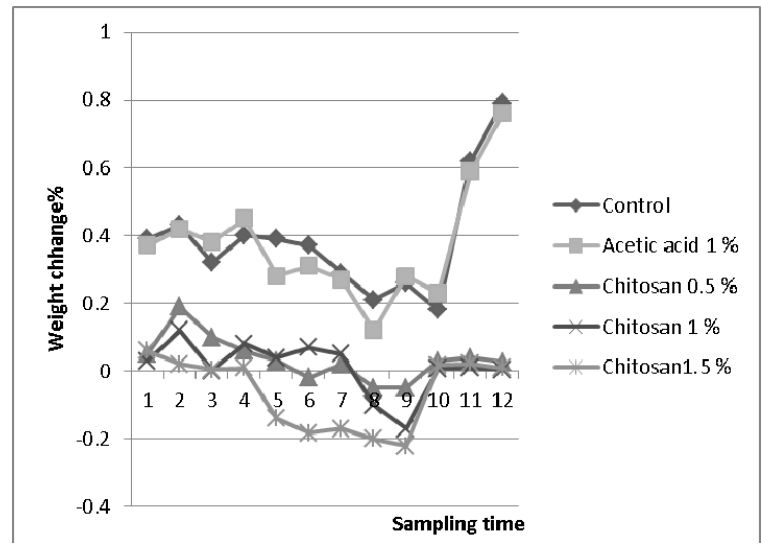

Fig 4. Chart of weight changes of pistachio nuts during 6 month storage.

\section{Chitosan effect on organolyptic specifications of Pistachio}

As a matter of fact that chitosan is of marine origin; from shrimp \& lobster waste, rarely it might create a fish or other marine products flavors, and influence on general acceptability; However, it might cause a bitter taste in some kind of products such as strawberry and lettuce. This comes back to the molecular weight and chitosan solution concentration and products combinations. Also, chitosan solution is colorless which does not affect the organoleptic properties of products. Tables 1 through 4 shows results from panel test of coated pistachio nuts [9].

1) Color: As it is evident from Fig.6, chitosan coating had no significant effect $(\mathrm{p}<0.05)$ on pistachio nuts color and maintain its color during storage; This result accords with Campanilo et al. findings in 2008 [4]. Photograph of the samples with and without chitosan coating showed in Fig 5; Its clear that there is no significant change in samples.
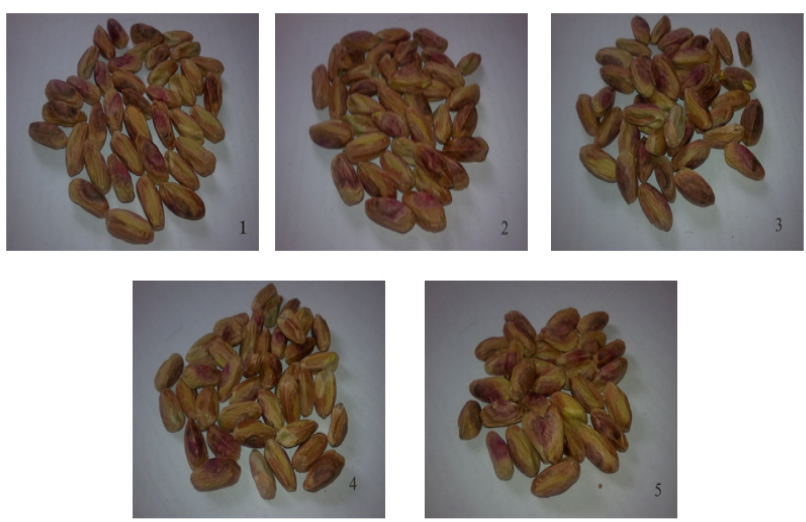

1)Control (sample without chitosan coating), 2)sample with acetic acid $1 \%$, 3) sample with chitosan $0.5 \%, 4$ ) sample with chitosan $1 \%, 5$ )sample with chitosan $1.5 \%$

Fig5. Photograph of the samples with and without chitosan coating

2) Flavor: Results related to the flavor (panel test) is showed in Fig. 7. As we can see $1.5 \%$ concentration of chitosan solution had a significant effect on pistachio nuts $(p<0.05)$. This might be due to higher concentration of chitosan solution rather than other treatments, in that with increasing chitosan concentration it causes a distinguishable marine product taste. The same result observed by Devlighere et al. in 2004 [9]. 


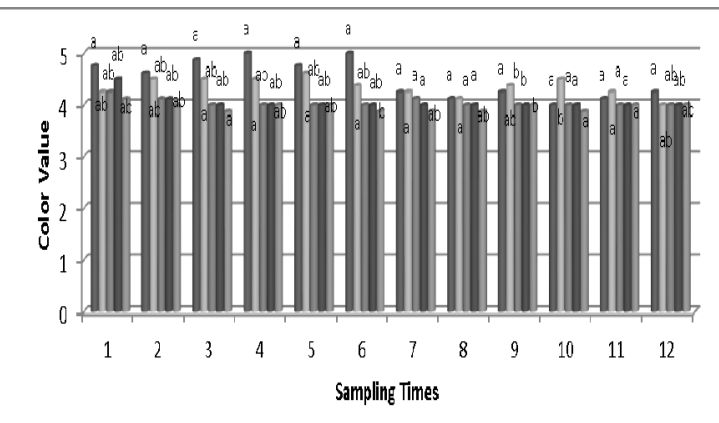

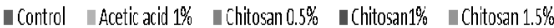

Fig6. Average comparison of different levels of chitosan effects on the color of pistachio nuts by hedonic-5-point test in the range of 1(bad) to 5 (very good). Numbers with the same letters in each sampling time: no significant difference.

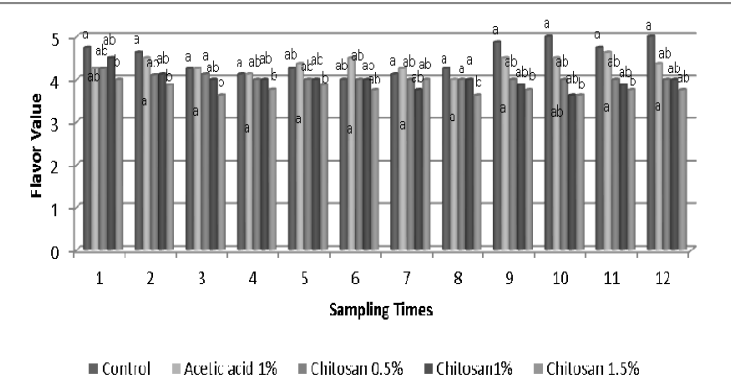

Fig. 7. Average comparison of different levels of chitosan effects on the flavor of pistachio nuts by hedonic-5-point test in the range of 1(bad) to 5 (very good). Numbers with the same letters in sampling time: no significant difference.

3) Texture: Fragility of pistachio nuts is an important factor which is significantly affected by moisture content. In this study, the humidity kept constant in all treatments in the range of control sample (about 4percent), which no significant difference were observed after comparison (p<0.05) (Fig. 8) [16].

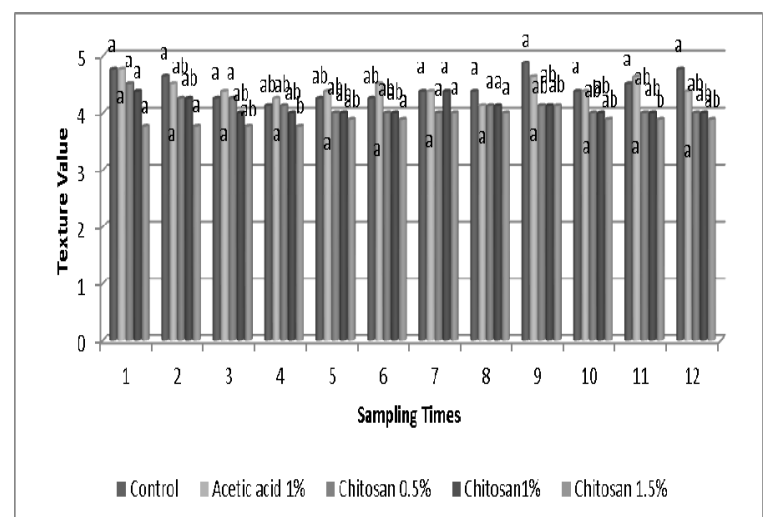

Fig. 8.. Average comparison of different levels of chitosan effects on the texture of pistachio nuts by hedonic-5-point test in the range of 1(bad) to 5 (very good). Numbers with the same letters in sampling time: no significant difference.

4) General acceptability: General acceptabilty was evaluated by Hedonic sensory evaluation. Comparing the average showed that pistachios general acceptability was not affected by different chitosan treatments $(\mathrm{p}<0.05)$.

Maximum quantity of general acceptability was 5 where the minimum quantity was about 4 Fig. 9. It means there was a little and insignificant change in organoleptic specifications of pistachio nuts treated with different concentrations of chitosan coating [11].

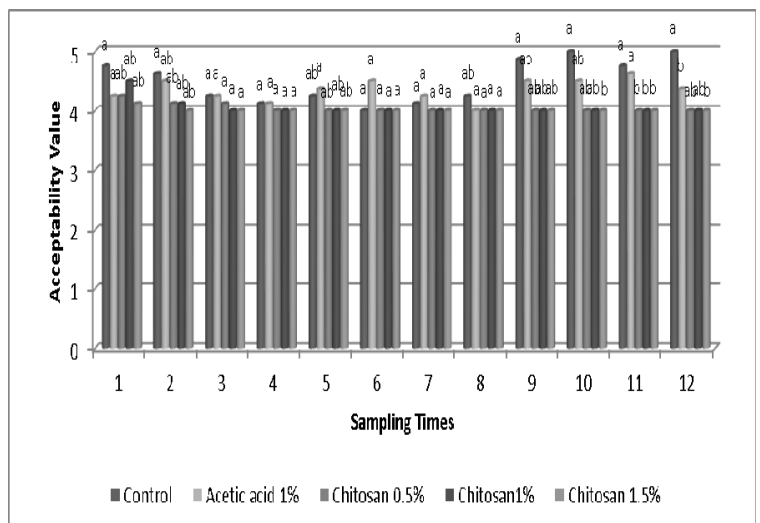

Fig. 9. Average comparison of different levels of chitosan effects on the general admission of pistachio nuts by hedonic-5-point test in the range of 1(bad) to 5 (very good). Numbers with the same letters in each sampling time: no significant difference.

\section{CONCLUSIONS}

As per the results obtained, chitosan would be a suitable coat for nuts such as pistachio. Concerning the economic point of view and antibacterial effect of chitosan, chitosan solution at $1 \%$ concentration is therefore recommended for coating purposes.

\section{REFERENCES}

[1] Koshteh, k. " Global pistachio production and marketing challenges." PhD Thesis ,university of Guelph ,Ontario, Canada. pp. 26-34, 2002.

[2] Synowiecki, J., Al-khateeb, N. "Production , properties, and some new applications of chitin and its derivatives." LWT-Food science and Technology. Vol. 43, pp. 14-5171, 2003.

[3] Kamil, J., Jeon, Y., Shahidi, F. "Antioxidative activity of chitosans of different viscosity in cooked comminuted flesh of herring". Food Chemistry. Vol. 79, pp.69-77, 2002.

[4] Campaniello, C. A., Bevilacqua, M., Sinigaglia, M. R. "Chitosan: Antimicrobial activity and potential applications for preserving minimally processed strawberries." Food Microbiology.Vol. 25, pp. 992-1000, 2008.

[5] Khalid, Z, BeatrizUrsúa, b., JuanI.Maté a. "Application of bioactive coatings based on chitosan for artichoke seed protection." Crop Protection. Vol. 29, pp. 853-859, 2010.

[6] Xianghong, M., Lingyu, Y., John, F., Kennedy, T. "Effects of chitosan and oligochitosan on growth of two fungal pathogens and physiological properties in pear fruit." Carbohydrate Polymers.Vol. 10, pp. 83-84, 2010

[7] Munoz, A., Moret, S., Garce, s. "Assessment of chitosan for inhibition of Colletotrichum sp. on tomatoes and grapes." Crop Protection. Vol. 28, pp. 36-40, 2009.

[8] Chien, P., Sheu, F., Lin, H. "Coating citrus (Murcott tangor) fruit with low molecular weight chitosan increases postharvest quality and shelf life." Food Chemistry. Vol. 100, pp. 1160-1164, 2007.

[9] Devlieghere, F., Vermeulen, A., Debevere, J., "Chitosan: antimicrobial activity, interactions with food components and applicability as a coating on fruit and vegetables." Food Microbiology.Vol. 21, pp.703-71, 2004.

[10] Dong, H., Cheng, L., Tan, J., Zheng, K., Jiang, Y. "Effects of chitosan coating on quality and shelf life of peeled litchi fruit." Journal of Food Engineering. Vol.64 , pp. 355-358. 2004.

[11] Cao , R., Xue, C., Liu, Q. "Changes in microbial flora of Pacific oysters (Crassostrea gigas) during refrigerated storage and its shelflife extension by chitosan." International Journal of Food Microbiology. Vol. 131, pp. 272-276. 2009.

[12] Badawy, E. I., Rabea, I." Potential of the biopolymer chitosan with different molecular weights to control postharvest gray mold of 
tomato fruit." Postharvest Biology and Technology. Vol. 51, pp.110-117, 2009.

[13] Bourtoom , T., chinnan, M. s. "Preparation and properties of ricestrarch- chitosan blend biodegradable film." LWT-Food science and Technology. Vol. 41, pp. 1633-1641, 2008.

[14] Costas G. Biliaderis, D., Marta, S., Izydorczyk, P. Functional Food Carbohydrates, CRC Press Taylor \& Francis Group, 2007.

[15] Belgheysi, S., Azizi, M., Zohurian, G., Hadian, Z. "Physical properties of whey protein edible films - Monoglyceride and coating effects on moisture loss and sensory characteristics of fresh mutton". Jurnal of Nutrition science and food science of Iran. Vol.3, pp. 83-93, 2009.

[16] Garez, H., Minaei, S., Basiri, A. "The effect of temperature, air velocity and thickness of product layer drying process of pistachio". Jurnal of Agricultural Science of Iran, Vol. 8, pp. 23-31, 2003.

[17] Kamangar, k. farrokhi, f. and Mehran, M. "Characterstics of pistachio kerneloil from Iranian cultivars". JAOCS. Vol. 52, pp. 512$513,1975$. 